$\mathrm{IMSc} / 2005 / 03 / 05$

\title{
On Energy Conditions and Stability in Effective Loop Quantum Cosmology
}

\author{
Golam Mortuza Hossain ${ }^{1, *}$ \\ ${ }^{1}$ The Institute of Mathematical Sciences, \\ CIT Campus, Chennai-600 113, India.
}

\begin{abstract}
In isotropic loop quantum cosmology, non-perturbatively modified dynamics of a minimally coupled scalar field violates weak, strong and dominant energy conditions when they are stated in terms of equation of state parameter. The violation of strong energy condition helps to have non-singular evolution by evading singularity theorems thus leading to a generic inflationary phase. However, the violation of weak and dominant energy conditions raises concern, as in general relativity these conditions ensure causality of the system and stability of vacuum via Hawking-Ellis conservation theorem. It is shown here that the non-perturbatively modified kinetic term contributes negative pressure but positive energy density. This crucial feature leads to violation of energy conditions but ensures positivity of energy density, as scalar matter Hamiltonian remains bounded from below. It is also shown that the modified dynamics restricts group velocity for inhomogeneous modes to remain sub-luminal thus ensuring causal propagation across spatial distances.

PACS numbers: 04.60.Pp, 04.60.Kz, 98.80.Jk
\end{abstract}

*Electronic address: golam@imsc.res.in 


\section{INTRODUCTION}

In general theory of relativity, dynamics of a spacetime is influenced by matter stressenergy tensor. Naturally, many properties regarding spacetime evolution can be concluded assuming some general properties of the matter stress-energy tensor, without having to know the details of the individual contributions from different matter sources. These requirements on the matter stress-energy tensor, widely called energy conditions, have been used to prove several important theorems in classical general relativity. One such theorem, the Hawking-Ellis conservation theorem [1, 2] says that if the matter stress-energy tensor is conserved, satisfies dominant energy condition and vanishes on a closed, achronal set $S$ then it also vanishes in the domain of dependence $D(S)$ of the set. Physically, this theorem ensures the stability of classical vacuum. As mentioned, the conservation theorem stands true provided the matter stress-energy tensor satisfies the dominant energy condition. This condition requires local energy density to be non-negative for all time-like observer and the energy-momentum 4-current to be non-spacelike i.e. the speed of energy-flow should not be exceeding the speed of light. Naturally, the violation of dominant energy condition raises concern about the causality and the stability of the system. However, it is worth pointing out that the above theorem does not have the converse i.e. although the dominant energy condition satisfying matter ensures causality and stability of the system but violation of this condition does not necessarily imply that the system violates causality or is unstable (see for example [3]). In such a situation, these issues should be considered for the specific context, as dominant energy condition violation and the Hawking-Ellis conservation theorem no longer vouch for the causality and the stability of the system.

In the cosmological context, the issue of dominant energy condition violation has acquired significant importance in recent literature. The observational evidences [4, 5] seem to suggest that in our universe major fraction of the energy density is contributed by some kind of mysterious dark energy that exerts negative pressure. The experimental data in this context not only allows but often favours the values of the equation of state parameter to be less than -1 for the dark energy component [6, 7, 8, 9, 10, 11, 12, 13, 14, 15, 16, 17]. Such values of the equation of state parameter require violation of dominant energy condition. This makes the problem of the dark energy even more severe which is otherwise itself a major theoretical challenge in the present day cosmology [18, 19, 20, 21, 22, 23]. A popular proposed model 
for dominant energy condition violating dark energy is so called phantom matter [6, 24, 25, 26, 27]. The phantom matter is essentially a minimally coupled scalar field model but with relatively negative kinetic term ( but see [28, 29, 30, 31, 32] for other possibilities). Naturally, the classical Hamiltonian for the phantom matter becomes unbounded from below. Such unbounded Hamiltonian essentially leads to a classically unstable system, as ground state of such system gets pushed to negative infinity.

Apart from the mentioned observational indication of violation of energy condition, there are in fact theoretical reasons to argue that some of these energy conditions in general relativity, should be violated in appropriate regime. One such reason behind this, is the existence of another important set of theorems, so called singularity theorems. These theorems tell us that if the evolution of a globally hyperbolic spacetime satisfies Einstein equation and the matter stress-tensor satisfies so called strong energy condition then the backward evolution of such an expanding spacetime is necessarily singular, in a sense that the spacetime is geodesically incomplete. In the cosmological context, it implies that if one considers the backward evolution of an expanding universe with respect to the coordinate time (lapse equal to unity) and the matter content satisfies strong energy condition then the physical quantities like energy density, spacetime curvature would diverge within finite time interval. However, the appearance of singularity in a classical theory is generally considered as an attempt to extrapolate the classical theory beyond its natural domain of validity, rather than considering it as a property of nature. Near the classical singularity one expects the evolution of the spacetime to be governed by a quantum theory of gravity, as classical description signals its own breakdown. Further, one also believes that a proper theory of quantum gravity should resolve the singularity that appears in the classical general relativity. Naturally, one would naively expect that the quantum effects of such theory should force the matter contents to effectively violate the strong energy condition when its dynamics is viewed as an evolution of pseudo-Riemannian spacetime.

In recent years the issues regarding singularities in cosmological models have been addressed in a rigorous way within the framework of loop quantum cosmology (LQC) 33, 34, 35, 36, 37, 38, 39, 40, 41]. The loop quantum cosmology is a quantization of the cosmological models along the line of a bigger theory known as loop quantum gravity (LQG) [42, 43, 44, 45]. It has been shown that the loop quantum cosmology cures the problem of classical singularities in isotropic model [46] as well as less symmetric homogeneous 
model [47] along with quantum suppression of classical chaotic behaviour near singularities in Bianchi-IX models [48, 49]. Further, it has been shown that non-perturbative modification of the scalar matter Hamiltonian leads to a generic phase of inflation [50, 51] (see 52] for related discussions on other kinds of matter). It has been also shown that loop quantum cosmology induced inflationary scenario can produce scale invariant primordial power spectrum as well as observed small amplitude for it, without fine tuning [53]. The primordial power spectrum contains a characteristic signature which is potentially falsifiable by observations. Further, it has been argued that the loop quantum cosmology induced inflationary phase can lead to a secondary standard inflationary phase [54, 55, 56, 57, 58]. This follows from the feature that the in-built inflationary period of loop quantum cosmology tends to produce favourable initial conditions for an additional standard inflationary phase. In [55], the authors have also studied the possible effects of the above mechanism on cosmic microwave background (CMB) angular power spectrum, generated during a standard inflationary phase that follows the loop quantum cosmology induced inflationary phase and shown that it can lead to suppression of power in the low CMB multipoles. These features crucially depends on a fact that in loop quantum cosmology the inverse triad (scale factor) operator [59] whose quantization relies on techniques used in full theory [60], has a bounded spectrum. This is unlike the classical situation where inverse scale factor blows up as scale factor goes to zero. However, not being a basic operator quantization of the inverse scale factor operator involves quantization ambiguities [61, 62].

From a quantum mechanical system, generally one obtains physically relevant quantities by computing physical expectation values of appropriate physical observables in the relevant physical states. In loop quantum cosmology, development of the machinery required to deal with them are still in nascent stage [36, 63, 64, 65]. Nevertheless, one can construct an effective but classical description of loop quantum cosmology using WKB techniques. The dynamics of the effective description is governed by an effective Hamiltonian [66] along with discreteness corrections [67, 68]. The effective loop quantum cosmology incorporates crucial non-perturbative modifications and has been shown to be generically non-singular as well 69, 70, 71, 72]. In fact several important features of loop quantum cosmology, that have been shown in literature, crucially rely on the effective classical description. Naturally, in the effective loop quantum cosmology, the violation of dominant energy condition raises serious concern. In particular, whether such effective classical description respects causality. 
In the cosmological context, any communication across spatial distances introduces inhomogeneity. So it is a natural concern to check whether the propagation of inhomogeneous modes respects causality. Also, whether such dominant energy condition violating effective description can ensure stability of the vacuum, as the Hawking-Ellis conservation theorem no longer guarantees for the same (see also [73, 74] for related discussions).

In section II, we briefly review the definitions of relevant energy conditions used in general relativity. In particular, for the cosmological context, we discuss the requirements on the equation of state parameter due to these energy conditions. In the next section, we discuss the properties of the equation of state parameters for a minimally coupled scalar field and also for the so-called phantom matter model of dark energy. In the section IV, we study the properties of the effective scalar matter Hamiltonian. In particular, we show that the kinetic term due to the modified dynamics, contributes negative pressure even though it contributes positive energy density. This crucial feature essentially leads to violation of dominant energy condition in terms of the equation of state parameter but it also ensures a bounded (from below) scalar matter Hamiltonian. In the next section, we derive a modified dispersion relation for the inhomogeneous modes due to the modified dynamics. Then we show that the group velocity for the relevant inhomogeneous modes remains sub-luminal thus ensuring causal propagation across spatial distances. We also compute the quantum corrections to the group velocity for a massless free scalar field at large volume.

\section{ENERGY CONDITIONS IN GENERAL RELATIVITY}

The energy conditions, often regarded as sacred principles [75], were mostly postulated to prove several important theorems in classical general relativity. A few important among them are the so called singularity theorems and conservation theorem. In this section, we will briefly recall the definitions of some of these energy conditions. In the cosmological context, these energy conditions can be essentially stated in terms of the energy density and its relation to the pressure component i.e. the equation of state parameter. We will mainly follow the convention of Wald [2]. 


\section{A. Weak Energy Condition}

For a given matter stress-energy tensor $T_{\mu \nu}$, the quantity $T_{\mu \nu} \xi^{\mu} \xi^{\nu}$ physically represents local energy density for an observer whose 4 -velocity is $\xi^{\mu}$ at a spacetime point. The weak energy condition is physically interpreted as the requirement of non-negativity for the classical energy density. Naturally, the weak energy condition is stated as

$$
T_{\mu \nu} \xi^{\mu} \xi^{\nu} \geq 0
$$

for all time-like $\xi^{\mu}$. Assuming that the stress-energy tensor can be diagonalized i.e. it can be written as $T_{\mu \nu}:=\rho t_{\mu} t_{\nu}+P_{1} x_{\mu} x_{\nu}+P_{2} y_{\mu} y_{\nu}+P_{3} z_{\mu} z_{\nu}$ where $\left\{t^{\mu}, x^{\mu}, y^{\mu}, z^{\mu}\right\}$ is an orthogonal set of basis and $t^{\mu}$ is time-like, the weak energy condition requires $\rho \geq 0$ and $\rho+P_{i} \geq 0$ for $i=1,2,3$ where $P_{i}$ is the principal pressure. For the homogeneous and isotropic spacetime these requirements can be conveniently stated in terms of the equation of state parameter $\omega:=P / \rho$ as $\omega \geq-1$ and the energy density $\rho \geq 0$.

\section{B. Strong Energy Condition}

A crucial requirement on the matter stress-energy tensor, for the singularity theorems to hold, is that it should satisfy so called strong energy condition. This energy condition requires matter stress-energy tensor to satisfy

$$
T_{\mu \nu} \xi^{\mu} \xi^{\nu} \geq-\frac{1}{2} T
$$

for all unit time-like $\xi^{\mu}$. Assuming diagonal form of the stress-energy tensor, the strong

energy condition requires $\rho+\sum_{j=1}^{3} P_{j} \geq 0$ and $\rho+P_{i} \geq 0$ for $i=1,2,3$. For the homogeneous and isotropic spacetime, these requirements in terms of the energy density and equation of state parameter can be stated as $\rho \geq 0, \omega \geq-\frac{1}{3}$. One may note here that the violation of strong energy condition which is necessary for non-singular cosmological evolution, implies an accelerating phase in its evolution via Raychaudhuri equation.

\section{Dominant Energy Condition}

The Hawking-Ellis conservation theorem requires matter stress-energy tensor to satisfy so called dominant energy condition. This condition requires the local energy density to be 
non-negative for all time-like observer and the local energy-momentum 4-current i.e $-T_{\mu \nu} \xi^{\mu}$ to be future directed, non-spacelike for all future directed, time-like $\xi^{\mu}$. So the dominant energy condition is stated as

$$
T_{\mu \nu} \xi^{\mu} \xi^{\nu} \geq 0 \quad ; \quad T_{\mu \nu} \xi^{\nu} T_{\rho}^{\mu} \xi^{\rho} \leq 0
$$

The second requirement can be physically interpreted as the requirement on matter stressenergy tensor such that the speed of energy-flow does not exceed the speed of light. Assuming diagonal form of the stress-energy tensor, the dominant energy condition requires $\rho \geq\left|P_{i}\right|$ for $i=1,2,3$. In other words, the energy density is required to dominate the pressure components. For the homogeneous and isotropic spacetime, these requirements can be stated in terms of the equation of state parameter as $|\omega| \leq 1$ and energy density $\rho \geq 0$.

Apart from the above energy conditions, there are few more energy conditions that can be seen in the literature. For example, so called null energy condition requires matter stressenergy tensor to satisfy $T_{\mu \nu} n^{\mu} n^{\nu} \geq 0$, for all null vector $n^{\mu}$.

\section{CLASSICAL SCALAR MATTER HAMILTONIAN}

In the cosmological scale, our universe appears to be spatially flat, homogeneous and isotropic with a very good precision. The invariant distance element in such spacetime (using natural units i.e. $c=\hbar=1$ ) is given by Friedmann-Robertson-Walker metric

$$
d s^{2}=-d t^{2}+a^{2}(t) d \mathbf{x}^{2},
$$

where $a(t)$ is the scale factor. Clearly, the metric components do not have any spatial dependence. In this paper we will consider a minimally coupled scalar field as the matter source. The dynamics of such scalar field is governed by the action

$$
S_{\phi}:=\int d^{4} x \sqrt{-g} \mathcal{L}=\int d^{4} x \sqrt{-g}\left[-\frac{1}{2} g^{\mu \nu} \partial_{\mu} \phi \partial_{\nu} \phi-V(\phi)\right] .
$$

Let us recall that we are mainly interested in studying the effects on the scalar field dynamics, due to the non-perturbative modification coming from loop quantum cosmology. In the canonical quantization, as in loop quantum cosmology, one treats Hamiltonian as a basic object that governs the dynamics of the system. Thus, for our purpose it is necessary to have the expression for the scalar matter Hamiltonian

$$
H_{\phi}=a^{-3} \int d^{3} x\left[\frac{1}{2} \pi_{\phi}^{2}\right]+a \int d^{3} x\left[\frac{1}{2}(\nabla \phi)^{2}\right]+a^{3} \int d^{3} x[V(\phi)],
$$


where field momentum density $\pi_{\phi}=a^{3} \dot{\phi}$. In deriving expression (6), it is assumed that the background geometry is homogeneous, isotropic and described by the metric (41). However, we have assumed that the scalar field itself need not be homogeneous. This approximation greatly simplifies the analysis. Nevertheless, one should keep it in mind that it is trustworthy as long as the deviation from homogeneity and isotropy remains small.

In loop quantum cosmology, the geometrical quantities like the scale factor $a$ here, are represented through corresponding quantum operators. While deriving effective classical Hamiltonian from loop quantum cosmology, these geometrical quantities effectively get replaced by the eigenvalues of their corresponding quantum operators. The kinetic term of the scalar matter Hamiltonian (6) involves inverse powers of the scale factor. In loop quantum cosmology, the inverse scale factor operator has a bounded spectrum. Clearly one can see that the kinetic term of the effective scalar matter Hamiltonian will involve non-perturbative modifications.

Given an arbitrary inhomogeneous scalar field in a spatially flat space, one can decompose it in terms its Fourier modes. In this case, the dynamics of the $k=0$ mode i.e. the spatially homogeneous mode will essentially drive the evolution of the homogeneous background geometry, as the contribution from non-zero $k$ modes are assumed to be small. So

for the purpose of determining the background evolution, it is sufficient to consider only the homogeneous mode. In other words, we will neglect the contribution from the gradient term while evaluating the background evolution. Naturally, the scalar matter Hamiltonian (6) reduces to

$$
H_{\phi}=p^{-\frac{3}{2}} \frac{1}{2} p_{\phi}^{2}+p^{\frac{3}{2}} V(\phi)
$$

where $\int d^{3} x \sqrt{-g}:=a^{3} V_{0}:=p^{\frac{3}{2}}$ and $p_{\phi}\left(=V_{0} \pi_{\phi}\right)$ is the field momentum. It is important to note here that we have absorbed the fiducial coordinate volume $V_{0}$ (of a given finite cell) in the definition of the variable $p$. In loop quantum cosmology, the variable $p$ is known as redefined densitized triad and it is one of the basic phase space variables.

\section{A. Classical Energy Density and Pressure}

In the Lagrangian formulation, one can obtain the expression for the general stress-energy tensor by considering the variation of the action with respect to the spacetime metric. Naturally, one can use the general expression of the stress-energy tensor, to obtain the 
reduced expression for the energy density and the pressure component for the homogeneous and isotropic spacetime. On the other hand, in the Hamiltonian formulation such direct method is not available. However, one can define the expression for the energy density and the pressure component in terms of the classical Hamiltonian as

$$
\rho:=\frac{1}{2} \dot{\phi}^{2}+V(\phi)=p^{-\frac{3}{2}} H_{\phi} \quad ; \quad P:=\frac{1}{2} \dot{\phi}^{2}-V(\phi)=-p^{-\frac{3}{2}}\left(\frac{2 p}{3} \frac{\partial H_{\phi}}{\partial p}\right) .
$$

It may be noted here that the definitions of the energy density and the pressure (8) in terms of the scalar matter Hamiltonian immediately ensure the matter conservation equation $\dot{\rho}=-3\left(\frac{\dot{a}}{a}\right)(\rho+P)$ along the classical trajectories.

\section{B. Classical Equation of State}

In the cosmological context, the equation of state parameter is defined as the ratio of the pressure component to the energy density as

$$
\omega:=\frac{P}{\rho}=\frac{\frac{1}{2} \dot{\phi}^{2}-V(\phi)}{\frac{1}{2} \dot{\phi}^{2}+V(\phi)}
$$

For a minimally coupled scalar field, the values of the equation of state parameter (9) are

restricted to be $|\omega| \leq 1$, as the scalar field $\phi$ takes values in the real line and the potential is required to satisfy $V(\phi) \geq 0$. In other words, the dynamics of a minimally coupled scalar field always respects the dominant energy condition. Thus, the Hawking-Ellis conservation theorem vouches for the stability of the ground state. The stability of the ground state can also be understood from the property of the scalar matter Hamiltonian (17). It is easy to see that the expression of the scalar matter Hamiltonian (7) ensures that it remains bounded from below. This property immediately implies a classically stable ground state for the system.

\section{Phantom Matter Equation of State}

As we have mentioned, in the phantom matter model of dark energy energy [6], one consider a minimally coupled scalar field but with relatively negative kinetic term. Thus, the energy density and the pressure component for the phantom field are given by

$$
\rho_{\text {Phantom }}:=-\frac{1}{2} \dot{\phi}^{2}+V(\phi) \quad ; \quad P_{\text {Phantom }}:=-\frac{1}{2} \dot{\phi}^{2}-V(\phi) .
$$


Clearly, the equation of state parameter for the phantom field $\omega_{\text {Phantom }}\left(:=P_{\text {Phantom }} / \rho_{\text {Phantom }}\right)$ can take value less than -1 . In other words, the phantom matter field violates the dominant energy condition. Naturally, the Hawking-Ellis conservation theorem does not guarantee for the stability of the ground state. In particular, using the corresponding Hamiltonian for the phantom matter, one can easily see that it no longer remains bounded from below. The unbounded (from below) Hamiltonian immediately implies that there does not exist a classically stable ground state for the system.

\section{EFFECTIVE SCALAR MATTER HAMILTONIAN}

In isotropic loop quantum cosmology, the basic phase space variables are Ashtekar connection and densitized triad. The geometrical property of the space is encoded in the densitized triad $p$ whereas the time variation of geometry is encoded in the connection. In loop quantum cosmology one redefines densitized triad to absorb the fiducial coordinate volume component. This makes the proper volume of the universe (44) to be $\int d^{3} x \sqrt{-g}=a^{3} V_{0}=p^{\frac{3}{2}}$ 40]. The effective scalar matter Hamiltonian for the classical system whose dynamics is governed by the Hamiltonian (7), is given by [66]

$$
H_{\phi}^{\mathrm{eff}}=\frac{1}{2}\left|\tilde{F}_{j, l}(p)\right|^{\frac{3}{2}} p_{\phi}^{2}+p^{\frac{3}{2}} V(\phi)
$$

where $\tilde{F}_{j, l}(p)$ is the eigenvalue of the inverse densitized triad operator $p^{-1}$ and is given by $\tilde{F}_{j, l}(p)=\left(p_{j}\right)^{-1} F_{l}\left(p / p_{j}\right)$ where $p_{j}=\frac{1}{3} \gamma \mu_{0} j l_{p}^{2}$. The $\mu_{0}$ is an order of unity parameter that appears while quantizing the Hamiltonian constraint operator in loop quantum cosmology [40]. The $j$ and $l$ are two quantization ambiguity parameters [61, 62]. The half integer $j$ is related with the dimension of representation while writing holonomy as multiplicative operator. The real valued $l(0<l<1)$ corresponds to different, classically equivalent ways of writing the inverse power of the densitized triad in terms of Poisson bracket of the basic variables. The function $F_{l}(q)$ is approximated as [49]

$$
\begin{aligned}
F_{l}(q)= & {\left[\frac { 3 } { 2 ( l + 2 ) ( l + 1 ) l } \left((l+1)\left\{(q+1)^{l+2}-|q-1|^{l+2}\right\}-\right.\right.} \\
& \left.\left.(l+2) q\left\{(q+1)^{l+1}-\operatorname{sgn}(q-1)|q-1|^{l+1}\right\}\right)\right]^{\frac{1}{1-l}} \\
\rightarrow & q^{-1} \quad(q \gg 1) \\
\rightarrow & {\left[\frac{3 q}{l+1}\right]^{\frac{1}{1-l}} \quad(0<q \ll 1) . }
\end{aligned}
$$


It is clear from the expression (12) that for the large values of the densitized triad i.e. in large volume one recovers the expected classical behaviour for the inverse densitized triad. The quantum behaviour is manifested for smaller values of the densitized triad. Here the meaning of large or small values of the triad $p$ is determined necessarily by the values of $p_{j}$. We will follow this convention throughout the paper unless explicitly stated.

\section{A. Effective Energy Density and Pressure}

In this paper, we are interested in studying the effects on the energy conditions due to the non-perturbative modification coming from loop quantum cosmology and its further implications. In the cosmological context, the energy conditions are stated in terms of the energy density and its relation to the pressure i.e. the equation of state parameter. In loop quantum cosmology, one obtains non-perturbative modification at the level of the effective Hamiltonian but not at the level of an effective action. This prevents one to directly obtain the expression of the effective stress-energy tensor. On the other hand, in classical general relativity the energy conditions are defined in terms of the stress-energy tensor. Naturally, the issue of energy conditions violation in the effective dynamics, is crucially related to the definitions of the effective energy density and pressure. In the classical situation we have seen that it is possible to write down the reduced standard expressions of the energy density and the pressure (8) purely in terms of the reduced Hamiltonian. These definitions of the energy density and the pressure immediately ensure the matter conservation equation along the classical trajectories. Naturally, one can use the same definitions for the effective energy density and the pressure just replacing the standard Hamiltonian in terms of the effective Hamiltonian. So we define the effective energy density and the effective pressure, following the definitions of classical energy density and pressure (마), as

$$
\rho^{\mathrm{eff}}:=p^{-\frac{3}{2}} H_{\phi}^{\mathrm{eff}} \quad ; \quad P^{\mathrm{eff}}:=-p^{-\frac{3}{2}}\left(\frac{2 p}{3} \frac{\partial H_{\phi}^{\mathrm{eff}}}{\partial p}\right) .
$$

It is worth pointing out that to define the effective energy density and the pressure, one could have proceeded as done in [66]. In this approach one first obtains the Hamilton's equations of motion for the matter degrees of freedom as well as the gravitational degrees of freedom. Then one rewrites these equations of motion, by suitable manipulations such that a part of these equations matches with the gravitational part of the standard Friedman equation and 
the Raychaudhuri equation. In the next step, one then reads off the expressions for effective energy density and the pressure by comparing with standard equations. These expressions of the energy density and the pressure agree with the definitions (13) when the contributions due to the non-perturbative modification of the gravity sector become negligible. Since the effective Hamiltonian description is strictly valid in the region where background geometry is essentially classical i.e. non-perturbative modification of geometry is negligible. Clearly, in such situation these two set of definitions agree with each other. It is important to emphasize here that although the non-perturbative modification of the gravity sector becomes negligible in the region of interest but the non-perturbative modification of the matter sector can still survive. In fact we are interested in studying the effects of non-perturbative modification of the scalar matter dynamics.

\section{B. Effective Equation of State}

Having known the expressions of the effective energy density and the pressure (13), one can easily define the effective equation of state parameter $\omega^{\text {eff }}:=P^{\text {eff }} / \rho^{\text {eff }}$. The evolution of the effective equation of state parameter depends on the effective Hamiltonian. However, as shown in [51], one can eliminate the explicit appearance of the effective Hamiltonian and can express the effective equation of state parameter in terms of the classical equation of state parameter $\omega$, as

$$
\omega^{\mathrm{eff}}=-1+\frac{(1+\omega) p^{\frac{3}{2}}\left[\tilde{F}_{j, l}(p)\right]^{\frac{3}{2}}\left(1-\frac{p}{\tilde{F}_{j, l}(p)} \frac{d \tilde{F}_{j, l}(p)}{d p}\right)}{(1+\omega) p^{\frac{3}{2}}\left[\tilde{F}_{j, l}(p)\right]^{\frac{3}{2}}+(1-\omega)} .
$$

Using the expression (12), it is easy to see that for the large values of the densitized triad $p$, where one expects the quantum effects to be small, $\omega^{\text {eff }} \simeq \omega$. On the other hand, for small values of $p, \omega^{\text {eff }}$ differs from the classical $\omega$ dramatically. Using the small volume (small triad) expression of the inverse densitized triad (12), one may note that the effective equation of state satisfies $\left(\omega^{\text {eff }}+1\right)<0$, for all allowed values of the ambiguity parameter $l$. Let's recall that in terms of equation of state parameter, the weak energy condition requires $(\omega+1) \geq 0$, the strong energy condition requires $\left(\omega+\frac{1}{3}\right) \geq 0$ and the dominant energy conditions requires $|\omega| \leq 1$. So it is clear that in loop quantum cosmology, the effective equation of state parameter violates all of these energy conditions due to the nonperturbative modifications. 


\section{Kinetic Contribution to Pressure}

The allowed values for the classical equation of state (9), are restricted to be $|\omega| \leq 1$. Naturally, it is an important question to ask how is it then possible for the effective equation of state to take values less than -1 , instead of the facts that in both cases one begins with a minimally coupled scalar field and uses the same definition for the equation of state parameter in terms of their corresponding Hamiltonian. The answer to this question lies in the fact that in effective loop quantum cosmology, although one begins with a standard minimally coupled scalar field but for small volume this coupling gets altered dramatically. The effective coupling remains minimal in a sense that it couples only through the geometrical variables but not through curvatures. However, it is clear that the gravity coupling to the scalar matter no longer remains standard minimal coupling as the spectrum of the inverse triad operator differs from the classical expression dramatically for small volume. To understand this issue better, let us have a look at the contributions due to the kinetic term to the pressure component $P_{\mathrm{KE}}$ for both cases

$$
P_{\mathrm{KE}}=-p^{-\frac{3}{2}}\left[\frac{p}{3} p_{\phi}^{2}\right] \frac{\partial}{\partial p}\left[p^{-\frac{3}{2}}\right] \quad ; \quad P_{\mathrm{KE}}^{\mathrm{eff}}=-p^{-\frac{3}{2}}\left[\frac{p}{3} p_{\phi}^{2}\right] \frac{\partial}{\partial p}\left[\left|\tilde{F}_{j, l}(p)\right|^{\frac{3}{2}}\right]
$$

It is evident from the equation (15) that in the standard case, the kinetic term contributes positive pressure. This is what one would intuitively expect from our understanding of ordinary thermo-dynamical system. However, in the effective loop quantum cosmology, using the expression of the inverse densitized triad (12), it is easy to see that the kinetic term contributes negative pressure for small volume even though for large volume it contributes positive pressure like in standard case. This crucial 'extra' negative pressure from the kinetic term is what essentially leads the effective equation of state to violate dominant energy condition. Clearly, the bounded spectrum of the inverse densitized triad plays a major role in this.

On the other hand, in the phantom matter model of dark energy, to obtain the values of the equation of state parameter to be less than -1 , one makes the kinetic term relatively negative by hand. This step essentially forces the kinetic term to contribute negative pressure. However, it also leads the kinetic term to contribute negative energy density. This step essentially jeopardise energy density expression as its positivity is no longer remain guaranteed. Clearly, a relatively negative kinetic term in the scalar matter Hamiltonian, makes it 
unbounded from below. In other words, the ground state of such system gets pushed to negative infinity. Naturally, naive quantization of such system can lead to a catastrophic decay of vacuum [25]. On contrary, in the effective loop quantum cosmology scenario, the kinetic term gives negative contribution only in the pressure expression but not in the energy density expression. Thus, although the equation of state parameter in effective loop quantum cosmology violates dominant energy condition but it also necessarily ensures the positivity of the energy density. It is also evident from the expression of the scalar Hamiltonian (11) that it remains bounded from below signifying a stable ground state.

\section{Example: Massive Scalar Field}

Now we take an explicit example to illustrate the dynamics of the scalar field at small volume regime where non-perturbative modification plays a significant role. For simplicity, we consider the dynamics of a massive free scalar field. In other words, the scalar potential is consist of only the mass term i.e. $V(\phi)=\frac{1}{2} m_{\phi}^{2} \phi^{2}$. To simplify further, we choose the value of the ambiguity parameter to be $l \rightarrow 0+$. With these assumptions the effective matter Hamiltonian for small volume becomes

$$
H_{\phi}^{\mathrm{eff}} \simeq p^{\frac{3}{2}}\left[\frac{1}{2}\left(3^{\frac{3}{2}} p_{j}^{-3}\right) p_{\phi}^{2}+\frac{1}{2} m_{\phi}^{2} \phi^{2}\right] .
$$

Using the Hamilton's equations of motion, one can obtain analytical solutions for the field equations, given by

$$
\phi=\sqrt{\frac{2 \bar{\rho}}{m_{\phi}^{2}}} \sin \left(\alpha p^{\frac{3}{2}}+c_{1}\right) ; p_{\phi}=\sqrt{\frac{2 \bar{\rho}}{\left(3^{\frac{3}{2}} p_{j}^{-3}\right)}} \cos \left(\alpha p^{\frac{3}{2}}+c_{1}\right),
$$

where $\alpha=\sqrt{\frac{\left(3^{\frac{3}{2}} p_{j}^{-3}\right)\left(m_{\phi}^{2}\right)}{24 \pi G \bar{\rho}}}, \bar{\rho}$ and $c_{1}$ are two constants of integration. Using the field solutions (17), one can easily see that along any trajectory $H_{\phi}^{\text {eff }} \simeq p^{\frac{3}{2}} \bar{\rho}$. One may note here that the energy density contribution due to the scalar field dynamics effectively looks a like contribution from a cosmological constant. The constant of integration $\bar{\rho}$ physically corresponds to the energy density during its evolution. This also implies an exponential inflationary phase. This is of course expected behaviour, as the effective equation of state parameter in loop quantum cosmology generically becomes $\omega^{\mathrm{eff}} \approx-1$ at small volume [51]. This simple example clearly shows that classical dynamics of the system is essentially stable, as we have argued for a general system with the modified scalar field dynamics. 


\section{PROPAGATION OF INHOMOGENEOUS MODES}

We have mentioned earlier that the second part of the dominant energy condition requires the speed of energy propagation not to exceed the speed of light. Naturally, the violation of dominant energy condition also raises the concern, whether such system can prohibit super-luminal flow of energy. In other words, whether such system can respect causality. In classical cosmology, one begins by postulating so called cosmological principle i.e. on large scale there is neither a preferred direction nor a preferred place in our universe. This principle is imposed by assuming that on cosmological scale our universe is spatially homogeneous and isotropic. The strict imposition of spatial homogeneity will prohibit any kind of spatial flow of energy as it will violate spatial homogeneity. However, this assumption undoubtedly is an idealisation and is made to rather simplify background dynamics. Naturally, if we want to allow some kind of spatial flow of energy then we must relax the spatial homogeneity. While relaxing this assumption nevertheless one should be careful so that we can still use the available machinery of the cosmological set-up. This is generally achieved by considering the deviation from spatial homogeneity to be small. It is worth pointing out that small spatial inhomogeneity in the matter field configuration will also lead to small inhomogeneity in the background geometry. For simplicity, however, we will treat the background geometry as homogeneous.

\section{A. Modified Klein-Gordon Equation}

We have seen earlier that the kinetic term of the scalar matter Hamiltonian gets nonperturbative modification, as its classical expression involves inverse powers of densitized triad. The effective scalar matter Hamiltonian, obtained as outlined in [53], is given by

$$
H_{\phi}^{\mathrm{eff}}=V_{0}\left|\tilde{F}_{j, l}(p)\right|^{\frac{3}{2}} \int d^{3} x\left[\frac{1}{2} \pi_{\phi}^{2}\right]+V_{0}^{-\frac{1}{3}} p^{\frac{1}{2}} \int d^{3} x\left[\frac{1}{2}(\nabla \phi)^{2}\right]+V_{0}^{-1} p^{3 / 2} \int d^{3} x[V(\phi)] .
$$

One may note here that we have now kept the gradient term in the effective Hamiltonian. The gradient term was neglected earlier while computing background evolution, as one assumes that the background evolution is mainly determined by the homogeneous and isotropic contribution of the matter Hamiltonian. It is worth pointing out here that the gradient term of the equation (18) having the correct sign, the corresponding dynamics does not suffer from the so called gradient instability [76, 77], another pathological feature of 
the phantom matter models. Using the Hamilton's equations of motion for the effective Hamiltonian (18), one can derive the corresponding modified Klein-Gordon equation

$$
\ddot{\phi}-3\left(\frac{p \tilde{F}_{j, l}^{\prime}(p)}{\tilde{F}_{j, l}(p)}\right)\left(\frac{\dot{a}}{a}\right) \dot{\phi}+\left|\tilde{F}_{j, l}(p)\right|^{\frac{3}{2}} p^{\frac{3}{2}}\left(-\frac{\nabla^{2} \phi}{a^{2}}+V^{\prime}(\phi)\right)=0,
$$

where $\tilde{F}_{j, l}^{\prime}(p) \equiv \frac{d \tilde{F}_{j, l}(p)}{d p}$. Using the expression for the spectrum of the inverse triad (12), it is easy to see that the modified Klein-Gordon equation (19) reduces to the standard Klein-Gordon equation at large volume.

In a given spatially flat spacetime background, an inhomogeneous scalar field can be decomposed in terms of its Fourier modes. The dynamics of the $k=0$ mode i.e. the spatially homogeneous mode essentially drives the evolution of the background geometry as the contributions from non-zero $k$ modes are assumed to be small. However, as we have argued that to study the energy propagation across spatial distance in the cosmological background, it is essential to consider the dynamics of inhomogeneous modes i.e. non-zero $k$ modes. The Fourier decomposition of the inhomogeneous scalar field is defined as

$$
\phi(\mathbf{x}, t)=\int \frac{d^{3} \mathbf{k}}{(2 \pi)^{3}}\left[\phi_{k}(t) e^{i \mathbf{k} \cdot \mathbf{x}}\right]
$$

where $\phi_{k}(t)$ are the Fourier components. For simplicity, we will consider the dynamics of a massless free scalar i.e. we will assume $V(\phi)=0$. Using the modified Klein-Gordon equation (19) and the equation (20), one can derive the modified equation for the Fourier modes

$$
\ddot{\phi}_{k}(t)-3\left(\frac{p \tilde{F}_{j, l}^{\prime}(p)}{\tilde{F}_{j, l}(p)}\right)\left(\frac{\dot{a}}{a}\right) \dot{\phi}_{k}(t)+\left|\tilde{F}_{j, l}(p)\right|^{\frac{3}{2}} p^{\frac{3}{2}}\left(\frac{k^{2}}{a^{2}}\right) \phi_{k}(t)=0 .
$$

In the small volume regime where the spectrum of the inverse triad operator can be approximated as $\tilde{F}_{j, l}(p) \sim p^{\frac{1}{1-l}}$ and the effective equation of state parameter as $\omega^{\text {eff }} \approx-1$, one can obtain an analytical solution for the equation (21) [53], given by

$$
\phi_{k}(t)=\eta^{\left(1+\frac{1}{2 n}\right)}\left[A_{(k, n)} J_{-\left(1+\frac{1}{2 n}\right)}(k \eta)+B_{(k, n)} J_{\left(1+\frac{1}{2 n}\right)}(k \eta)\right]
$$

where $J_{n}(x)$ are the Bessel functions, $A_{(k, n)}$ and $B_{(k, n)}$ are two constants of integration corresponding to second order differential equation. The variable $\eta$ is defined as $d \eta:=a^{-n} d t$, where the parameter $n=-\frac{1}{2}\left(1+\frac{3}{1-l}\right)$. In loop quantum cosmology allowed values for the ambiguity parameter $l$ is $(0<l<1)$. Naturally, the new parameter $n$ takes values as 
$(-\infty<n<-2)$. The argument of the Bessel function $k \eta$ can be conveniently expressed in terms the scale factor as $k \eta=\frac{k}{H a}\left(\frac{a^{1-n}}{-n}\right)$, where $H\left(\equiv \frac{\dot{a}}{a}\right)$ is the Hubble parameter.

At first let's study the large wavelength $(k \rightarrow 0)$ behaviour of the general solution (22). For the general solution (22) when both constants of integration $A_{(k, n)}$ and $B_{(k, n)}$ are present then using the asymptotic form of the Bessel function $J_{m}(x) \approx \frac{1}{\Gamma(1+m)}\left(\frac{x}{2}\right)^{m}$ for $x<<1$, it is easy to see that $\phi_{k}(t)$ becomes approximately constant and becomes proportional to $A_{(k, n)}$. For the special case when $A_{(k, n)}$ is identically zero then $\phi_{k}(t)$ remains time dependent but its time dependence is non-oscillatory. These features of the Fourier modes $\phi_{k}(t)$ can also be seen directly from the differential equation (21). For the larger wavelength modes the third term in the equation (21) can be neglected. The approximated second order differential equation then admits a constant solution and a non-oscillatory time-dependent solution, as expected. Clearly, the second term which is a (anti)friction term, plays a major role for the larger wavelength modes. Since, our main interest is to study the energy transmission across spatial distances then clearly the larger wavelength modes are not relevant for this purpose. On the other hand, for smaller wavelength $(k \rightarrow \infty)$ modes, the general solution become oscillatory, as the asymptotic form of the Bessel function is $J_{m}(x) \approx \sqrt{\frac{2}{\pi x}} \cos \left(x-\frac{m \pi}{2}-\frac{\pi}{4}\right)$ for $x>>1$. Naturally, the smaller wavelength modes are the potential carriers for the energy transmission across spatial distances. For smaller wavelength modes, the effects of the (anti)friction term is negligible. Thus, for simplicity we will neglect the (anti)friction term in the equation (21) for further analysis. The information regarding assumed small inhomogeneity are encoded in the amplitudes of the mode functions $\phi_{k}(t)$. Since propagation speed of linear waves does not depend on their amplitudes, the causal properties of the propagating inhomogeneous modes are quite insensitive to the exact details of their amplitudes.

\section{B. Modified Dispersion Relation}

In the cosmological context, any spatial transmission of energy will introduce inhomogeneity. So to investigate causality of the system, it is natural to study the group velocity for the inhomogeneous modes. One may recall that in a medium where absorption (friction) or emission (anti-friction) is small, the group velocity essentially determine the speed of signal propagation [78]. To compute the group velocity it is convenient to find out the relation between its frequency and wave-number i.e. the dispersion relation. Using the governing 
equation for the inhomogeneous modes (21), neglecting the (anti)friction term, and making the ansatz $\phi_{k}(t) \sim e^{i \tilde{\omega} t}$, one can easily derive the modified dispersion relation in effective loop quantum cosmology as

$$
\tilde{\omega}^{2} \approx\left|\tilde{F}_{j, l}(p)\right|^{\frac{3}{2}} p^{\frac{3}{2}}\left(\frac{k^{2}}{a^{2}}\right) .
$$

In the classical situation 'inverse triad' is just the inverse of triad i.e $p \times \tilde{F}_{j, l}(p)=1$. The dispersion relation (23) then becomes same as the standard Minkowskian dispersion relation between frequency and physical wave number $(k / a)$. In loop quantum cosmology the spectrum of the inverse triad operator is bounded. Hence the dispersion relation in effective loop quantum cosmology differs dramatically for small volume compared to the standard dispersion relation.

It is worth emphasising that the modification in the dispersion relation that is being studied here, arises because of the bounded spectrum of the inverse triad. This modification is distinct from the different types of modification generally considered in the literature. For example, in the context of quantum gravity scenario [79, 80, 81, 82, 83] or in the context of trans-Planckian inflationary scenario [84, 85], one considers modification of standard dispersion relation by introducing appropriate non-linearity.

\section{Group velocity}

It is worthwhile to emphasize here that the group velocity determines the speed of signal propagation only if the absorption or amplification of the signal remains small. In other words, 'signal transmission' makes sense only if the original signal reaches its target without major distortion while propagation (see 78] for related discussion). In the effective loop quantum cosmology scenario, we have argued that the relevant modes for energy transmission across spatial distances, are the smaller wavelength modes and for these modes the (anti)friction term plays very little role in their evolution. Using the dispersion relation (23), one can easily compute the group velocity for the inhomogeneous modes as

$$
v_{g}:=\frac{d \tilde{\omega}}{d(k / a)}=\left|\tilde{F}_{j, l}(p)\right|^{\frac{3}{4}} p^{\frac{3}{4}} .
$$

In the classical situation right hand side of the expression (24) is identically equal to unity. Physically, this implies that for the massless free scalar field, the inhomogeneous modes 
transmit signals at the speed of light. However, in the effective loop quantum cosmology it is no longer the case. Using the expression for the spectrum of the inverse triad (12), it is easy to see that in the small volume regime, the speed of signal propagation is in fact much slower than the speed of light (in classical vacuum). The group velocity for the inhomogeneous modes gradually increases and approaches the speed of light towards the end of the non-perturbatively modified dynamics.

It is worth emphasising here that the actual spectrum of the inverse scale factor operator is fundamentally non-differentiable. However, to study the qualitative consequences of it within an effective analysis, one uses a peace-wise analytic function $F_{l}(q)$ (12) which approximates the spectrum of the inverse scale factor operator. This is a good approximation provided the scale $p_{j}$ is sufficiently large. However, being peace-wise analytic this approximation is good as long as $\left(p<<p_{j}\right)$ or $\left(p>>p_{j}\right)$ but not near the transition regime, as the approximation function $F_{l}(q)(12)$ is not analytic at $q=1\left(q=p / p_{j}\right)$. So the governing equation of the mode functions (21) which involves $F_{l}(q)$ as well as its derivative, is not defined near $p=p_{j}$. Thus, the derivation and the subsequent expression of the group velocity (24) are valid as long as $\left(p<<p_{j}\right)$ or $\left(p>>p_{j}\right)$ but not in the neighbouring regime of $p=p_{j}$. However, there still exist a significant small volume regime even excluding the regime near $p=p_{j}$, as the validity of approximation for the spectrum of the inverse scale factor operator, requires $p_{j}$ to be large.

Thus, in effective loop quantum cosmology although non-perturbatively modified dynamics violates dominant energy condition in terms of the equation of state parameter but the underlying modified dynamics restricts the group velocity for the inhomogeneous modes to remain sub-luminal. In the cosmological context we have argued that any spatial transmission of energy will introduce spatial inhomogeneity. Here in the effective loop quantum cosmology, we have shown that the group velocity for the inhomogeneous modes remains sub-luminal due to the non-perturbative modification. Clearly, in effective loop quantum cosmology, non-perturbatively modified dynamics of a minimally coupled scalar field respects causality. The violation of dominant energy condition is essentially dictated by the $k=0$ mode but this mode is not relevant for the purpose of signal transmission across spatial distances.

It is worth pointing out that the 'speed of light' here is meant to imply the speed of electromagnetic wave propagation in the classical vacuum that determines the causal struc- 
ture of the spacetime. This is important to emphasize because in loop quantum cosmology, one expects to get similar non-perturbative modification even to the electromagnetic wave propagation. Then the actual speed of light in the effective loop quantum cosmology itself may become slower compared to the speed of light in the classical vacuum. Intuitively, one may consider the small volume effective background geometry, coming from loop quantum cosmology, as a refractive medium with a value of the group index $n_{g}\left(\equiv c / v_{g}\right)$ is greater than unity. In this context, the group index is same as the refractive index, as the phase velocity is same as the group velocity.

\section{QUANTUM CORRECTIONS TO GROUP VELOCITY AT LARGE VOLUME}

Using the spectrum of the inverse triad operator (12), it is easy to see that although at large volume the leading term is just the inverse of triad but there are sub-leading terms also in its expression. Naturally, in the effective loop quantum cosmology, the group velocity for the inhomogeneous modes is not exactly equal to unity even at the large volume. Using the expression (12), for a massless free scalar field, one can compute the group velocity with quantum corrections as

$$
v_{g} \simeq\left[1+\frac{3(2-l)}{40}\left(\frac{p_{j}^{2}}{p^{2}}\right)\right] .
$$

It is clear from the expression (25) that the corrections to the group velocity at large volume is extremely small but positive as $(0<l<1)$. The group velocity becomes equal to unity as the volume of the system goes to infinity. To have some numerical estimate of this finite volume quantum correction, let us choose say $p_{j} \sim 10^{5} l_{p}^{2}$. The observed size of universe today is $\sqrt{p} \sim 10^{60} l_{p}$. Then the correction to the group velocity due to modified spectrum of the inverse triad operator, today is $\sim 10^{-231}$ ! It is extremely unlikely that such small correction will have any significant effect. Even for the cosmological context (time scale $\sim 10^{17} \mathrm{sec}$ ) such small deviation of group velocity, may be completely irrelevant.

\section{DISCUSSIONS}

To summarize, in effective loop quantum cosmology, non-perturbatively modified dynamics of a minimally coupled scalar field violates weak, strong and dominant energy conditions when they are stated in terms of equation of state parameter. The violation of strong energy 
condition although helps to have non-singular evolution by evading the singularity theorems but the violation of weak and dominant energy conditions raises concern. In classical general relativity, these energy conditions are used to prohibit super-luminal flow of energy and to ensure the stability of classical vacuum via the Hawking-Ellis conservation theorem. Naturally, the violation of these energy conditions in terms of effective equation of state parameter, raises concern about the causality and the stability of the system. In this paper, we have shown that although at face value these energy conditions are violated, underlying modified dynamics in effective loop quantum cosmology nevertheless ensures positivity of energy density, as scalar matter Hamiltonian remains bounded from below. Considering the modified dynamics for the inhomogeneous modes, we have shown that group velocity for the relevant modes remains sub-luminal in small volume regime, thus ensuring causal propagation across spatial distances. We have also computed the large volume quantum corrections to the group velocity of the inhomogeneous modes for the massless free scalar field.

Now, let us try to understand the physical phenomena behind this rather unusual feature of the non-perturbatively modified dynamics. In the case of classical dynamics of a minimally coupled scalar field, the values of the equation of state parameter are restricted to be $|\omega| \leq 1$. However, in the case of modified dynamics, the effective equation of state can take values less than -1 . This is rather surprising given the facts that one begins with a minimally coupled scalar field and uses the same definition of equation of state for both the cases. This 'anomalous' behaviour follows from the fact that at the small volume, non-perturbatively modified gravity becomes repulsive although it remains attractive for the large volume. This feature can be easily seen by considering a classical trajectory of a massless free scalar field. The non-perturbatively modified scalar matter Hamiltonian, along any trajectory, increases with the increasing scale factor for small volume but decrease for large volume. Naturally, the gravitational Hamiltonian, to satisfy the Hamiltonian constraint $\left(H_{\phi}+H_{\text {grav }}=0\right)$, must decrease with increasing scale factor for small volume. Later, in the large volume it starts increasing with increasing scale factor. This immediately implies that modified gravitational interaction is repulsive for small volume whereas for large volume, as one expects, it is attractive. This repulsive nature of the gravitational interaction manifest itself through the non-standard gravity coupling to the scalar matter Hamiltonian via bounded spectrum of the inverse triad operator. 
For a standard minimally coupled scalar field, the kinetic term contributes positive pressure. Of course, this is what one would intuitively expect from our understanding of ordinary thermo-dynamical system. However, in the effective loop quantum cosmology, the kinetic term contributes negative pressure for small volume even though for large volume it contributes positive pressure like in standard case. This crucial 'extra' negative pressure from the kinetic term is what essentially leads the effective equation of state to violate dominant energy condition. Clearly, the bounded spectrum of the inverse densitized triad plays a major role in this. On the other hand, in phantom matter model of dark energy, to obtain the values of the equation of state parameter to be less than -1 , one makes the kinetic term relatively negative by hand. This change of sign essentially forces the kinetic term to contribute negative pressure. However, it also leads the kinetic term to contribute negative energy density. This step badly affects the energy density expression, as its positivity is no longer certain. In other words, a relatively negative kinetic term in the scalar matter Hamiltonian, makes it unbounded from below. This implies that the system does not have a stable classical ground state. On contrary, in the effective loop quantum cosmology scenario, the kinetic term gives negative contribution only in the pressure expression but not in the energy density expression. Thus, although the equation of state parameter in effective loop quantum cosmology violates dominant energy condition but it necessarily ensures the positivity of the energy density. It is also evident from the expression of the scalar Hamiltonian (11) that it remains bounded from below, signifying a stable classical ground state.

The bounded spectrum of the inverse triad (scale factor) operator plays the central role in violating the energy conditions. The violation of energy conditions although leads to a generic inflationary phase and allows to have a non-singular evolution but it also makes the causality and the stability of the system uncertain. However, as shown in this paper, the same bounded spectrum in fact acts as a saviour to ensure the causality and the stability of the system. It is worth pointing out that the quantization of the inverse triad was not invented to obtain the bounded spectrum such that these physical features follow. Rather it was quantized following the techniques used in the full theory of loop quantum gravity. The quantization of the inverse triad involves ambiguities but these crucial features are insensitive to their precise values. It may be worth emphasising that although the exercise presented here is not directly related with the dark energy scenario, one may learn an important lesson from here that if one wants to construct a dominant energy condition violating yet 
well behaved scalar field model of dark energy then one should look beyond the standard minimal coupling.

It is now important to discuss some subtleties of the analysis presented here. In classical general relativity, the definitions of the energy conditions are generally covariant. However, in the cosmological context, the energy conditions are stated with respect to a preferred frame namely the so-called comoving frame. Thus, one must be careful while interpreting

the results in more general context. Secondly, in the Lagrangian formulation one obtains the reduced expression of the energy density and pressure for the homogeneous and isotropic spacetime, using a generally covariant expression of the stress-energy tensor. In the Hamiltonian formulation such a spacetime covariant method is not available. Naturally, one needs to define the expression of energy density and pressure, in terms of the scalar matter Hamiltonian. In the classical situation although they are equivalent but with the non-trivial quantum corrections this issue is rather subtle. In the analysis presented here, we have assumed the background geometry as homogeneous although we have allowed the scalar field living in it to become inhomogeneous. This approximation is trustworthy as long as the deviation from the homogeneity remains sufficiently small. Further, we have considered the non-perturbative modification of the kinetic term only. Using slightly different quantization strategy, one could obtain a factor of 'triad times inverse triad' also in the gradient term. However, such modification would change only the quantitative nature of the results shown here but not the qualitative nature. Naturally, the features of the non-perturbatively modified dynamics shown here, are robust under this quantization ambiguity.

\section{Acknowledgments}

I thank Ghanashyam Date and Martin Bojowald for careful reading, critical comments and insightful suggestions on the manuscript. I thank Ghanashyam Date, Parampreet Singh, Romesh Kaul for helpful, illuminating discussions.

[1] S.W. Hawking and G.F.R. Ellis, "The Large Scale Structure of Space-time", Cambridge University Press, 1973.

[2] Robert M. Wald, "General Relativity", University of Chicago Press, 1984. 
[3] B. McInnes, "The dS/CFT correspondence and the big smash," JHEP 0208, 029 (2002) arXiv:hep-th/0112066.

[4] A. G. Riess et al. [Supernova Search Team Collaboration], "Observational Evidence from Supernovae for an Accelerating Universe and a Cosmological Constant," Astron. J. 116, 1009 (1998) arXiv:astro-ph/9805201.

[5] S. Perlmutter et al. [Supernova Cosmology Project Collaboration], "Measurements of Omega and Lambda from 42 High-Redshift Supernovae," Astrophys. J. 517, 565 (1999) arXiv:astro-ph/9812133.

[6] R. R. Caldwell, "A Phantom Menace?," Phys. Lett. B 545, 23 (2002) arXiv:astro-ph/9908168.

[7] A. Melchiorri, L. Mersini-Houghton, C. J. Odman and M. Trodden, "The State of the Dark Energy Equation of State," Phys. Rev. D 68, 043509 (2003) arXiv:astro-ph/0211522.

[8] U. Alam, V. Sahni, T. D. Saini and A. A. Starobinsky, "Is there Supernova Evidence for Dark Energy Metamorphosis?," Mon. Not. Roy. Astron. Soc. 354, 275 (2004) arXiv:astro-ph/0311364.

[9] T. R. Choudhury and T. Padmanabhan, "A theoretician's analysis of the supernova data and the limitations in determining the nature of dark energy II: Results for latest data," Astron. Astrophys. 429, 807 (2005) arXiv:astro-ph/0311622.

[10] Y. Wang and P. Mukherjee, "Model-Independent Constraints on Dark Energy Density from Flux-averaging Analysis of Type Ia Supernova Data," Astrophys. J. 606, 654 (2004) arXiv:astro-ph/0312192.

[11] S. Nesseris and L. Perivolaropoulos, "A comparison of cosmological models using recent supernova data," Phys. Rev. D 70, 043531 (2004) arXiv:astro-ph/0401556.

[12] Y. G. Gong, "Supernova constraints on dark energy model," arXiv:astro-ph/0401207

[13] U. Alam, V. Sahni and A. A. Starobinsky, "The case for dynamical dark energy revisited," JCAP 0406, 008 (2004) arXiv:astro-ph/0403687.

[14] Y. Wang and M. Tegmark, "New dark energy constraints from supernovae, microwave background and galaxy clustering," Phys. Rev. Lett. 92, 241302 (2004) arXiv:astro-ph/0403292.

[15] B. Feng, X. L. Wang and X. M. Zhang, "Dark Energy Constraints from the Cosmic Age and Supernova," Phys. Lett. B 607, 35 (2005) arXiv:astro-ph/0404224.

[16] H. K. Jassal, J. S. Bagla and T. Padmanabhan, "WMAP constraints on low redshift evolution 
of dark energy," Mon. Not. Roy. Astron. Soc. 356, L11 (2005) arXiv:astro-ph/0404378.

[17] P. S. Corasaniti, M. Kunz, D. Parkinson, E. J. Copeland and B. A. Bassett, "The foundations of observing dark energy dynamics with the Wilkinson Microwave Anisotropy Probe," Phys. Rev. D 70, 083006 (2004) arXiv:astro-ph/0406608.

[18] S. Weinberg, "The Cosmological Constant Problem," Rev. Mod. Phys. 61, 1 (1989).

[19] S. M. Carroll, W. H. Press and E. L. Turner, "The Cosmological constant," Ann. Rev. Astron. Astrophys. 30, 499 (1992).

[20] V. Sahni and A. A. Starobinsky, "The Case for a Positive Cosmological Lambda-term," Int. J. Mod. Phys. D 9, 373 (2000) arXiv:astro-ph/9904398.

[21] S. M. Carroll, "The cosmological constant," Living Rev. Rel. 4, 1 (2001) arXiv:astro-ph/0004075.

[22] P. J. E. Peebles and B. Ratra, "The cosmological constant and dark energy," Rev. Mod. Phys. 75, 559 (2003) arXiv:astro-ph/0207347.

[23] T. Padmanabhan, "Cosmological constant: The weight of the vacuum," Phys. Rept. 380, 235 (2003) arXiv:hep-th/0212290.

[24] A. E. Schulz and M. J. White, "The tensor to scalar ratio of phantom dark energy models," Phys. Rev. D 64, 043514 (2001) arXiv:astro-ph/0104112.

[25] S. M. Carroll, M. Hoffman and M. Trodden, "Can the dark energy equation-of-state parameter w be less than -1?," Phys. Rev. D 68, 023509 (2003) arXiv:astro-ph/0301273.

[26] P. Singh, M. Sami and N. Dadhich, "Cosmological dynamics of phantom field," Phys. Rev. D 68, 023522 (2003) arXiv:hep-th/0305110.

[27] M. Sami and A. Toporensky, "Phantom Field and the Fate of Universe," Mod. Phys. Lett. A 19, 1509 (2004) arXiv:gr-qc/0312009.

[28] V. K. Onemli and R. P. Woodard, "Super-acceleration from massless, minimally coupled $\phi^{4}$," Class. Quant. Grav. 19, 4607 (2002) arXiv:gr-qc/0204065.

[29] S. Nojiri and S. D. Odintsov, "Quantum deSitter cosmology and phantom matter," Phys. Lett. B 562, 147 (2003) arXiv:hep-th/0303117.

[30] E. Elizalde, S. Nojiri and S. D. Odintsov, "Late-time cosmology in (phantom) scalartensor theory: Dark energy and the cosmic speed-up," Phys. Rev. D 70, 043539 (2004) arXiv:hep-th/0405034.

[31] V. K. Onemli and R. P. Woodard, "Quantum effects can render w $<-1$ on cosmological scales," 
Phys. Rev. D 70, 107301 (2004) arXiv:gr-qc/0406098.

[32] S. M. Carroll, A. De Felice and M. Trodden, "Can we be tricked into thinking that w is less than -1?," Phys. Rev. D 71, 023525 (2005) arXiv:astro-ph/0408081.

[33] M. Bojowald, "Loop quantum cosmology. I: Kinematics," Class. Quant. Grav. 17, 1489 (2000) arXiv:gr-qc/9910103.

[34] M. Bojowald, "Loop quantum cosmology. II: Volume operators," Class. Quant. Grav. 17, 1509 (2000) arXiv:gr-qc/9910104.

[35] M. Bojowald, "Loop quantum cosmology. III: Wheeler-DeWitt operators," Class. Quant. Grav. 18, 1055 (2001) arXiv:gr-qc/0008052.

[36] M. Bojowald, "Loop quantum cosmology. IV: Discrete time evolution," Class. Quant. Grav. 18, 1071 (2001) arXiv:gr-qc/0008053.

[37] M. Bojowald, "Isotropic loop quantum cosmology," Class. Quant. Grav. 19, 2717 (2002) arXiv:gr-qc/0202077.

[38] M. Bojowald, "The semiclassical limit of loop quantum cosmology," Class. Quant. Grav. 18, L109 (2001) arXiv:gr-qc/0105113.

[39] M. Bojowald and H. A. Morales-Tecotl, "Cosmological applications of loop quantum gravity," Lect. Notes Phys. 646, 421 (2004) arXiv:gr-qc/0306008.

[40] A. Ashtekar, M. Bojowald and J. Lewandowski, "Mathematical structure of loop quantum cosmology," Adv. Theor. Math. Phys. 7, 233 (2003) arXiv:gr-qc/0304074.

[41] M. Bojowald, "The Early Universe in Loop Quantum Cosmology," arXiv:gr-qc/0503020.

[42] C. Rovelli, "Loop quantum gravity," Living Rev. Rel. 1, 1 (1998) arXiv:gr-qc/9710008.

[43] T. Thiemann, "Introduction to modern canonical quantum general relativity," arXiv:gr-qc/0110034.

[44] T. Thiemann, "Lectures on loop quantum gravity," Lect. Notes Phys. 631, 41 (2003) arXiv:gr-qc/0210094.

[45] A. Ashtekar and J. Lewandowski, "Background independent quantum gravity: A status report," Class. Quant. Grav. 21, R53 (2004) arXiv:gr-qc/0404018.

[46] M. Bojowald, "Absence of singularity in loop quantum cosmology," Phys. Rev. Lett. 86, 5227 (2001) arXiv:gr-qc/0102069.

[47] M. Bojowald, "Homogeneous loop quantum cosmology," Class. Quant. Grav. 20, 2595 (2003) arXiv:gr-qc/0303073. 
[48] M. Bojowald and G. Date, "Quantum suppression of the generic chaotic behavior close to cosmological singularities," Phys. Rev. Lett. 92, 071302 (2004) arXiv:gr-qc/0311003.

[49] M. Bojowald, G. Date and G. M. Hossain, "The Bianchi IX model in loop quantum cosmology," Class. Quant. Grav. 21, 3541 (2004) arXiv:gr-qc/0404039.

[50] M. Bojowald, "Inflation from quantum geometry," Phys. Rev. Lett. 89, 261301 (2002) arXiv:gr-qc/0206054.

[51] G. Date and G. M. Hossain, "Genericness of inflation in isotropic loop quantum cosmology," Phys. Rev. Lett. 94, 011301 (2005) arXiv:gr-qc/0407069.

[52] P. Singh, "Effective state metamorphosis in semi-classical loop quantum cosmology," arXiv:gr-qc/0502086.

[53] G. M. Hossain, "Primordial density perturbation in effective loop quantum cosmology," arXiv:gr-qc/0411012.

[54] M. Bojowald and K. Vandersloot, "Loop quantum cosmology, boundary proposals, and inflation,” Phys. Rev. D 67, 124023 (2003) arXiv:gr-qc/0303072.

[55] S. Tsujikawa, P. Singh and R. Maartens, "Loop quantum gravity effects on inflation and the CMB," Class. Quant. Grav. 21, 5767 (2004) arXiv:astro-ph/0311015.

[56] M. Bojowald, J. E. Lidsey, D. J. Mulryne, P. Singh and R. Tavakol, "Inflationary cosmology and quantization ambiguities in semi-classical loop quantum gravity," Phys. Rev. D 70, 043530 (2004) arXiv:gr-qc/0403106.

[57] J. E. Lidsey, D. J. Mulryne, N. J. Nunes and R. Tavakol, "Oscillatory universes in loop quantum cosmology and initial conditions for inflation," Phys. Rev. D 70, 063521 (2004) arXiv:gr-qc/0406042 .

[58] D. J. Mulryne, N. J. Nunes, R. Tavakol and J. E. Lidsey, "Inflationary cosmology and oscillating universes in loop quantum cosmology," arXiv:gr-qc/0411125.

[59] M. Bojowald, "The inverse scale factor in isotropic quantum geometry," Phys. Rev. D 64, 084018 (2001) arXiv:gr-qc/0105067.

[60] T. Thiemann, "QSD V: Quantum gravity as the natural regulator of matter quantum field theories," Class. Quant. Grav. 15, 1281 (1998) arXiv:gr-qc/9705019.

[61] M. Bojowald, "Quantization ambiguities in isotropic quantum geometry," Class. Quant. Grav. 19, 5113 (2002) arXiv:gr-qc/0206053.

[62] M. Bojowald, "Loop quantum cosmology: Recent progress," Pramana 63, 765 (2004) 
arXiv:gr-qc/0402053.

[63] G. M. Hossain, "Hubble operator in isotropic loop quantum cosmology," Class. Quant. Grav. 21, 179 (2004) arXiv:gr-qc/0308014.

[64] M. Bojowald, P. Singh and A. Skirzewski, "Time dependence in quantum gravity," arXiv:gr-qc/0408094

[65] K. Noui, A. Perez and K. Vandersloot, "On the physical Hilbert space of loop quantum cosmology," arXiv:gr-qc/0411039.

[66] G. Date and G. M. Hossain, "Effective Hamiltonian for isotropic loop quantum cosmology," Class. Quant. Grav. 21, 4941 (2004) arXiv:gr-qc/0407073.

[67] K. Banerjee and G. Date, "Discreteness corrections to the effective Hamiltonian of isotropic loop quantum cosmology," arXiv:gr-qc/0501102.

[68] K. Vandersloot, "On the Hamiltonian constraint of loop quantum cosmology," arXiv:gr-qc/0502082,

[69] P. Singh and A. Toporensky, "Big crunch avoidance in $\mathrm{k}=1$ loop quantum cosmology," Phys. Rev. D 69, 104008 (2004) arXiv:gr-qc/0312110.

[70] G. V. Vereshchagin, "Qualitative approach to semi-classical loop quantum cosmology," JCAP 0407, 013 (2004) arXiv:gr-qc/0406108.

[71] G. Date and G. M. Hossain, "Genericness of big bounce in isotropic loop quantum cosmology," Phys. Rev. Lett. 94, 011302 (2005) arXiv:gr-qc/0407074.

[72] D. J. Mulryne, R. Tavakol, J. E. Lidsey and G. F. R. Ellis, "An emergent universe from a loop," arXiv:astro-ph/0502589.

[73] D. H. Coule, "Contrasting quantum cosmologies," arXiv:gr-qc/0312045.

[74] D. H. Coule, "Quantum cosmological models," arXiv:gr-qc/0412026.

[75] B. Carter, "Energy dominance and the Hawking-Ellis vacuum conservation theorem," arXiv:gr-qc/0205010.

[76] S. D. H. Hsu, A. Jenkins and M. B. Wise, "Gradient instability for wi-1," Phys. Lett. B 597, 270 (2004) arXiv:astro-ph/0406043.

[77] R. V. Buniy and S. D. H. Hsu, "Instabilities and the null energy condition," arXiv:hep-th/0502203

[78] L. Brillouin, "Wave Propagation and Group Velocity," Academic Press, New York and London, 1960. 
[79] M. Bojowald, H. A. Morales-Tecotl and H. Sahlmann, "On loop quantum gravity phenomenology and the issue of Lorentz invariance," arXiv:gr-qc/0411101

[80] R. Gambini and J. Pullin, "Nonstandard optics from quantum spacetime," Phys. Rev. D 59, 124021 (1999) arXiv:gr-qc/9809038.

[81] J. Alfaro, H. A. Morales-Tecotl and L. F. Urrutia, "Quantum gravity corrections to neutrino propagation," Phys. Rev. Lett. 84, 2318 (2000) arXiv:gr-qc/9909079.

[82] J. Alfaro, H. A. Morales-Tecotl and L. F. Urrutia, "Loop quantum gravity and light propagation," Phys. Rev. D 65, 103509 (2002) arXiv:hep-th/0108061.

[83] H. Sahlmann and T. Thiemann, "Towards the QFT on curved spacetime limit of QGR. II: A concrete implementation," arXiv:gr-qc/0207031.

[84] J. Martin and R. H. Brandenberger, "The trans-Planckian problem of inflationary cosmology," Phys. Rev. D 63, 123501 (2001) arXiv:hep-th/0005209.

[85] J. C. Niemeyer, "Inflation with a high frequency cutoff," Phys. Rev. D 63, 123502 (2001) arXiv:astro-ph/0005533. 